\title{
European guidelines for quality assurance in colorectal cancer screening and diagnosis. First Edition Executive summary
}

Authors

Institutions
L. von Karsa' ${ }^{1}$ J. Patnick ${ }^{2}$, N. Segnan ${ }^{3}$

Quality Assurance Group, Early Detection and Prevention Section, International Agency for Research on Cancer, Lyon, France 2 Oxford University Cancer Screening Research Unit, Cancer Epidemiology Unit, University of Oxford, Oxford, United Kingdom \& NHS Cancer Screening Programmes, Sheffield, United Kingdom

3 CPO Piemonte and S. Giovanni University Hospital, Turin, Italy
Keywords

- mass screening

- colorectal neoplasms

- population-based programme

- screening process

- evidence-based guidelines
Bibliography

Dol http://dx.doi.org/ $10.1055 / \mathrm{s}-0032-1309822$

Endoscopy 2012; 44: SE1-SE8

(C) Georg Thieme Verlag KG

Stuttgart $\cdot$ New York

ISSN 0013-726X

Corresponding author

\section{L. von Karsa}

Quality Assurance Group

Early Detection and Prevention

Section

International Agency for

Research on Cancer

150 cours Albert Thomas

69372 Lyon Cedex 08

France

karsal@iarc.fr

qas@iarc.fr
Multidisciplinary, evidence-based guidelines for quality assurance in colorectal cancer screening and diagnosis have been developed by experts in a project coordinated by the International Agency for Research on Cancer. The full guideline document covers the entire process of populationbased screening. It consists of 10 chapters and over 250 recommendations, graded according to the strength of the recommendation and the supporting evidence. The 450-page guidelines and the extensive evidence base have been published

\section{Background}

$\nabla$

According to the most recent estimates by the International Agency for Research on Cancer [10] colorectal cancer (CRC) is the most common cancer in Europe with 432000 new cases in men and women reported annually. It is the second most common cause of cancer deaths in Europe with 212000 deaths reported in 2008. Worldwide CRC ranks third in incidence and fourth in mortality with an estimated 1.2 million cases and 0.6 million deaths annually.

In the 27 Member States of the European Union, CRC ranks first in incidence and second in mortality with approximately 334000 new cases and 149000 deaths estimated for men and women combined in 2008 [10]. Even in those Member States in the lower range of age-standardised rates of CRC, the burden of disease is significant compared to other regions of the world (see [10]). CRC is therefore an important health problem across the European Union (EU)

Screening is an important tool in cancer control in countries with a significant burden of CRC, provided the screening services are of high quality [35]. The EU recommends population-based screening for breast, cervical and colorectal cancer using evidence-based tests with quality assurance of the entire screening process including diagnosis and management of patients with screen- by the European Commission. The content of the executive summary is presented here to promote international discussion and collaboration by making the principles and standards recommended in the new EU Guidelines known to a wider professional and scientific community. Following these recommendations has the potential to enhance the control of colorectal cancer through improvement in the quality and effectiveness of screening programmes and services.

detected lesions [9]. The presently reported multidisciplinary, evidence-based guidelines for quality assurance in colorectal cancer screening and diagnosis have been developed by experts and published by the EU [28].

\section{Results}

\section{$\nabla$}

A large number of guiding principles and over 250 specific recommendations graded according to the supporting evidence and the strength of the respective recommendation are presented in 10 chapters. The scope of the 450-page guidelines document is summarized below.

\section{Role of screening in colorectal cancer control}

The aim of screening is to lower the burden of cancer in the population by discovering disease in its early latent stages. This permits more effective treatment than if diagnosed later when symptoms occur. Early treatment of invasive lesions, for example by endoscopic resection of early CRC, can be generally less detrimental for quality of life. The endoscopic removal of pre-malignant lesions also reduces the incidence of CRC by stopping the progression to cancer. Randomised trials in people of average risk invited to attend 
screening have shown a reduction in CRC mortality $[4,12,14,20]$ and incidence $[4,19]$.

\section{Council Recommendation on cancer screening}

The potential of screening for improving control of CRC has been recognised by the Council of the European Union. On 2 December 2003 the Council recommended implementation of populationbased screening programmes using evidence-based tests for breast, cervical and colorectal cancer to the EU Member States [9]. The Council Recommendation fulfils the criteria for screening defined by the World Health Organization [36] and takes into account the substantial experience in implementation of population-based cancer screening programmes in the EU. The Recommendation spells out fundamental principles of best practice in early detection of cancer. It invites EU Member States to take common action to implement cancer screening programmes with an organised, population-based approach and appropriate quality assurance at all levels, taking into account European quality assurance Guidelines for cancer screening, where they exist [34].

By the end of 2007, ten EU Member States were in the process of implementing national population-based CRC screening programmes (Cyprus, Finland, France, Hungary, Italy, Poland, Portugal, Romania, Slovenia and the United Kingdom) [7]. Furthermore, seven Member States had established nationwide nonpopulation-based programmes. In the meantime, 12 Member States have newly established or have upgraded the status of their existing CRC screening programmes (Czech Republic, Denmark, France, Ireland, Lithuania, the Netherlands, Portugal, Slovakia, Slovenia, Spain, Sweden and the United Kingdom).

\section{Need for effective quality assurance}

The potential harm caused by CRC screening includes the creation of unnecessary anxiety and morbidity, inappropriate economic cost, and exposure to the risk of invasive procedures for detection and diagnosis as well as for removal of lesions detected in screening. As demonstrated in implementation of breast and cervical cancer screening programmes, overall screening outcome and quality depend on the performance at each step in the screening process. To achieve the potential benefit of CRC screening, quality must therefore be optimal at each step in the process. This includes identification and personal invitation of the target population, performance of the screening test and, if necessary, diagnostic work-up, treatment, surveillance and aftercare of screen-detected lesions $[1,25,35]$.

Screening is performed on predominantly healthy people; comprehensive quality assurance is also required to maintain an appropriate balance between benefit and harm in the large numbers of people eligible to attend cancer screening programmes. The Council of the European Union therefore recommends appropriate, comprehensive quality standards and best practice in the implementation of cancer screening programmes. European quality assurance Guidelines for breast and cervical cancer screening have been developed by experts and published by the EU $[2,26]$. The availability of the new European guidelines for quality assurance in colorectal cancer screening and diagnosis will now make similar standards available to the Member States in which colorectal cancer screening programmes are currently running or being established.
Primary screening test recommended by the EU

The Council Recommendation calls for introduction of new cancer screening tests in routine healthcare only after they have been evaluated in randomised controlled trials (RCTs). To date, only the faecal occult blood test (FOBT) for men and women aged 50-74 years has been recommended by the EU for CRC screening [9]. In addition, any screening policy for colorectal cancer should take into account the available evidence and the numerous other principles and standards of best practice laid down in the Council Recommendation. Although the use of endoscopic screening methods is increasing, the majority of colorectal cancer screening examinations performed in the EU use the evidence-based test recommended by the Council of the EU.

\section{Purpose of the EU quality assurance Guidelines}

The purpose of the new EU Guidelines is not to recommend other modalities that might currently also be suitable for CRC screening in the EU. Instead, the Guidelines provide guiding principles and evidence-based recommendations on the quality assurance that should be followed when implementing screening programmes using the various modalities currently adopted in publicly mandated CRC screening programmes in the Member States.

The Editors have been conscious of the importance of raising and maintaining quality standards across all the EU Member States. While never abandoning those standards and recommendations that are crucial for mortality reduction, we have as far as possible attempted to achieve an equitable balance that can be used across a wide spectrum of cultural and economic healthcare settings. As with any standards and recommendations, these should be continuously reviewed in the light of future experience. It is not the purpose of these guidelines to promote recent research findings before they have been demonstrated to be of proven benefit in clinical practice. Neither should this edition be regarded as a textbook or in any way a substitute for practical clinical training and experience.

The Guidelines have been developed to inform European policymakers and public health specialists, and any other interested parties about the essential issues, guiding principles, standards and procedures of quality assurance and best practice that should be taken into account in running and establishing colorectal cancer screening programmes in the EU Member States.

The Guidelines have been specifically developed for screening of the average-risk population in which most CRC develops. Highrisk individuals should be referred for high-risk protocols if available. Since the relative variation in the moderate risk of developing CRC in most people with a family history of CRC is less than the geographic variation in average risk between the Member States, no attempt was made to develop recommendations tailored to this subgroup of the population. However, in the absence of hereditary syndromes people identified with a family history of CRC should not be excluded from average risk screening (see Chapter 2 [18]). The potential benefit and harm of screening recommendations tailored to people with a positive family history could be examined in greater depth in the preparation of the next edition of the Guidelines.

\section{Process of guideline development}

The Guidelines have been developed in an international collaborative project that was co-financed by the EU Public Health Programme. The project involved over 90 experts serving as authors, contributors, editors or reviewers from 32 countries including $21 \mathrm{EU}$ Member States 13 of which acceded to the EU be- 
fore 2004 (Austria, Belgium, Denmark, Finland, France, Germany, Italy, Luxembourg, Portugal, Spain, Sweden, The Netherlands and the United Kingdom) and eight of which acceded later to the EU (Czech Republic, Hungary, Latvia, Lithuania, Malta, Poland, Romania and Slovenia), as well as one EU applicant country (Croatia). The other countries represented among the collaborators included Argentina, Australia, Canada, China, India, Israel, Japan, Korea, Norway and the United States of America.

The new EU quality assurance Guidelines build on the successful developments in previous editions of the other EU screening Guidelines. The comprehensive CRC Guidelines cover the entire screening process from invitation to management of screen-detected lesions. Although the Guidelines focus on elements essential to screening, it is recognised that certain principles are equally important in diagnosis. Training, multi-disciplinary teamwork, monitoring and evaluation, cost-effectiveness, minimising adverse effects, and timeliness of further investigations are referred to repeatedly throughout the chapters. The applicability of many of the recommended standards and procedures to quality assurance in both screening and diagnosis is therefore reflected in the title of the first edition. Variations in style and emphasis have been unavoidable given the diverse sources of the contributions. However, the editors have maintained a high degree of conformity of approach.

The process used for identifying and evaluating the relevant evidence and for developing respective recommendations in the new Guidelines is described in detail elsewhere in this supplement [22]. Briefly, scientific and editorial management was provided by an editorial board with extensive experience in development of best practice guidelines, in evaluation of strategies for CRC screening and in programme management. The editorial board drafted an initial comprehensive outline of the Guidelines and recruited a multidisciplinary group of experts from across Europe to collaborate in revising the outline and drafting the chapters of the guideline according to an agreed methodology.

Additional scientific support was provided by a Literature Group consisting of epidemiologists with special expertise in the field of CRC and in critical appraisal of clinical studies. The Literature Group worked closely with the authors and editors in preparing and conducting systematic reviews of the literature on clinical questions of key importance. Bibliographic searches for most clinical questions were limited to the years 2000 to 2008 and were performed on Medline, and in many cases also on Embase and the Cochrane Library. Additional searches were conducted without date restrictions or starting before 2000 if the authors or editors who were experts in the field knew that there were relevant articles published before 2000. Articles of adequate quality recommended by authors because of their clinical relevance were also included. Preliminary versions of the draft guidelines were repeatedly reviewed and revised through multidisciplinary meetings of the authors, editors and the Literature Group, as well as in pan-European network meetings with participants from all of the EU Member States.

\section{Guideline publication format}

The print version of the Guidelines ( 450 pages) consists of 10 chapters each of which includes a list of key recommendations at the beginning of the chapter. The recommendations are graded according to the strength of the recommendation and the supporting evidence (for scale see below). The respective evidence is also summarised in the body of the chapters, with explicit cita- tion of over 750 references in the Guidelines. In total over 250 recommendations are provided.

The version of the Guidelines provided on the internet (web version) includes all of the elements in the print version, as well as an extensive Appendix 1 in digital format (1000 pages) with a complete record of the key clinical questions and corresponding bibliographic searches conducted by the Literature Group. The search results are documented in table format, and in summary documents. Altogether summary documents for over 100 clinical questions, and over 500 evidence tables are provided [21].

The level of evidence and the strength of each of the key recommendations presented in the front of each chapter is indicated using the following grading scales:

For the level of evidence:

I multiple randomised controlled trials (RCTs) of reasonable sample size, or systematic reviews (SRs) of RCTs

II one RCT of reasonable sample size, or 3 or less RCTs with small sample size

III prospective or retrospective cohort studies or SRs of cohort studies; diagnostic cross-sectional accuracy studies

IV retrospective case-control studies or SRs of case-control studies, time-series analyses

v case series; before/after studies without control group, cross-sectional surveys

VI expert opinion

For the strength of the respective recommendation:

A intervention strongly recommended for all patients or targeted individuals

B intervention recommended

C intervention to be considered but with uncertainty about its impact

D intervention not recommended

E intervention strongly not recommended

Images illustrating the chapter on Quality assurance in pathology in colorectal cancer screening and diagnosis are provided on a virtual pathology website at: http://www.virtualpathology.leeds.ac. uk.

\section{Scope of recommendations in the Guideline chapters}

The numerous guiding principles, evidence-based recommendations and conclusions presented in the new EU Guidelines for quality assurance in colorectal cancer screening and diagnosis cannot all be presented here. In addition to the key aspects of screening policy and methodology already mentioned above, the following points are highlighted in order to illustrate the scope and depth of the recommendations and conclusions in the first edition.

\section{Chapter 1 - Evidence for the effectiveness of colorectal cancer screening}

The first chapter [15] deals with the currently available evidence for the effectiveness of CRC screening, key operational parameters (age-range, interval between two negative screening examinations, or some combinations of tests) and cost-effectiveness. Among other things, the discussion of the 17 graded recommendations presented in the chapter reveals that the most evidence is available for the primary screening test (FOBT) recommended by the EU. 
Chapter 2 - Organisation of colorectal screening programmes

The 29 recommendations and conclusions in Chapter 2 [18] deal with key organisational aspects that influence the quality and effectiveness of CRC screening. There is a broad consensus in the EU on the fundamental principle that a colorectal cancer screening programme is a multidisciplinary undertaking. The effectiveness of the programme is a function of the quality of the individual components of the process.

It is also recognised that the provision of the screening service must account for the values and preferences of individuals as well as the perspectives of public health. The public health perspective in the planning and provision of screening services requires commitment to ensuring equity of access and sustainability of the programme over time. Taking into account the perspective of the individual requires commitment to promoting informed participation and to providing a high quality, safe service. Successful implementation of a screening programme entails more than simply carrying out the screening tests and referring individuals to assessment whenever indicated. Specific protocols must also be developed for identifying and subsequently inviting the target population. Protocols are also required for patient management in the diagnosis, treatment, and surveillance phases in order to ensure that all individuals have timely access to the proper diagnostic and treatment options.

Irrespective of the organisational approach, it should be recognised that appropriate political and financial support is crucial to the successful implementation of any screening programme.

\section{Chapter 3 - Evaluation and interpretation of screening outcomes}

Chapter 3 [23] includes 20 graded recommendations on the processes and procedures required for effective monitoring and evaluation of CRC screening programmes. Of fundamental importance is the complete and accurate recording of all relevant data on each individual and every screening test performed - including the test results, the decisions made as a consequence, diagnostic and treatment procedures and the subsequent outcome, including cause of death.

The chapter also provides an overview of performance measurements currently available from published trial results and population-based screening programmes. Based on this evidence and experience in implementation of population-based screening programmes, the authors and editors were able to reach a consensus on recommended standards of acceptable and desirable performance for a number of parameters. These initial standards, as well as the relevant standards available from other chapters are presented in a table at the end of the Executive Summary. The numbering of the standards is not indicative of importance. As explained elsewhere in the Guidelines, programmes should monitor numerous additional parameters in order to maintain and continuously improve quality. It is hoped that adherence to the other recommendations in the Guidelines will lead to development of a database that permits future expansion and improvement of the current standards.

\section{Chapter 4 - Faecal occult blood testing}

Chapter 4 [11] includes 21 detailed and in some cases complex recommendations dealing with design and application of faecal occult blood tests in CRC screening. It is recognised that the ideal biochemical test for population-screening of colorectal cancer would use a biomarker, specific and sensitive for both cancer and pre-cancer, on an easily collected sample, that could be safely and cheaply transported to a centralised laboratory for accurate, reproducible, and inexpensive automated analysis. In addition to these factors which are important for test performance, other key aspects should be taken into account that may influence the acceptability of the test in the target population. These include the design of the test kit, the instructions provided with the kit and the manner in which it is distributed. Laboratory quality assurance and external quality assessment also play an important role.

\section{Chapter 5 - Quality assurance in endoscopy}

Chapter 5 [32] provides a comprehensive view of the many-faceted aspects of quality assurance in endoscopy in its use both for the follow-up of screen-positives as well as for primary screening. ${ }^{1}$ The complexity of the relevant issues is reflected by the comparatively large number of specific recommendations dealing with planning and location of endoscopic services, infrastructure and equipment, preparation of the patient and aftercare, endoscopic technique, performance of endoscopists, quality improvement, policies and processes; a total of 50 recommendations.

The organisation of the chapter follows the patient journey to provide an explanation of the relevant issues of quality assurance that can also be used to improve the acceptability of CRC screening. This approach reflects the fundamental consensus of the authors and editors that everyone undergoing endoscopy, whether for primary screening, for assessment of abnormalities detected in screening, for assessment of symptoms, or for surveillance, should have as pleasant an experience as possible. A positive experience will help encourage people to recommend screening, assessment and surveillance to their friends, family and colleagues.

It is also recognised that the screening service must take into account the perspectives of endoscopy as well as public health to ensure that the experience is high-quality, safe and efficient as well as person-oriented. Furthermore, screening should take account of historic developments within different local and cultural contexts.

Although primary screening endoscopy is less complex than follow-up endoscopy (of screen-positives) primarily because of the lower frequency of high-risk lesions in primary screening endoscopy, care must be taken to ensure that the introduction of screening does not compromise endoscopy services for symptomatic patients and that screening and symptomatic (diagnostic) services achieve the same minimum levels of quality and safety. It is also recognised that, wherever possible, the quality assurance required for screening should have an enhancing effect on the quality of endoscopy performed for symptomatic patients and for other reasons. As for the other chapters in these Guidelines, the authors of Chapter 5 [32] have emphasised that screening and diagnosis of appropriate quality requires a multidisciplinary approach to diagnosis and management of lesions detected during endoscopy.

\section{Chapter 6 - Professional requirements and training}

Chapter 6 [31] provides 23 graded recommendations dealing with the requisite competency of screening staff. As previously mentioned with regard to the other chapters in the Guidelines,

\footnotetext{
${ }^{1}$ Note that although endoscopic screening programmes are running in some Member States, the FOBT is the only CRC screening test currently recommended by the EU [9].
} 
the fundamental need for a multidisciplinary approach and hence the need for special training of the multidisciplinary team that is responsible for a colorectal screening programme is recognised.

All staff involved in the delivery of a colorectal cancer screening programme require knowledge of the basic principles of colorectal cancer screening. The need for specialist training in screening differs between the different disciplines and is most important for those involved in the delivery of the service and diagnosis, e. g. laboratory staff, endoscopists, radiologists, pathologists and nurses. The surgical treatment of screen-detected cancer and post-operative treatment is not performed differently according to whether a cancer is screen detected or symptomatic, but there are certain considerations for the surgeon to take into account when treating a screen-detected cancer. Professional requirements of oncologists are not discussed in this chapter because, stage for stage, their role in the treatment of screen-detected disease is no different from that in symptomatic disease.

\section{Chapter 7 - Quality assurance in pathology}

Chapter 7 [27] provides practical guidelines for pathology within a colorectal screening programme. The pathology service plays a very important role in colorectal cancer screening since the management of participants in the programme depends on the quality and accuracy of the diagnosis. Pathology affects the decision to undergo further local and/or a major resection as well as surveillance after screening. The adoption of formal screening programmes leads to improvement not only in the management of early but also of advanced disease through the introduction of guidelines, quality standards, external quality assurance and audit. In screening programmes, the performance of individuals and programmes must be assessed and it is advantageous if common diagnostic standards are developed to ensure quality, recognise areas where sufficient evidence is still lacking, and initiate high-quality studies to gather the evidence required.

The chapter includes 23 graded recommendations concentrating on the areas of clinical importance [27]. It is hoped that these recommendations will also help to standardise quality and performance across the European Union. The associated annex deals with some of the more difficult areas and suggests topics for future research [33]. Guidelines for the reporting and management of resected specimens have been included in an attempt to move towards agreed minimum European standards of pathology in these areas as well. This is the first edition of what will be a continuing process of revision as new data emerge on the pathology, screening and management of colorectal cancer. It is also hoped that by setting minimum standards, these will be followed in all programmes and that this will encourage the development of higher standards amongst the pathology community and screening programmes.

\section{Chapter 8 - Management of lesions detected in colorectal cancer screening}

The inclusion of a chapter with 32 graded recommendations on management of lesions detected in CRC screening [30] recognises that reduction in CRC mortality is the main endpoint of any CRC screening programme. It is also recognised that all screening modalities will detect substantial numbers of individuals with adenomas [17] as well as a lesser number of lesions in the serrated pathway, some of which should be treated as adenomas (see Ch. 7 [27]). As adenomas are recognised to be pre-malignant [16] screening has the potential to reduce the incidence of the disease if these lesions are adequately managed. To achieve the dual aims of mortality and incidence reduction it is essential that all the elements of the screening service achieve and maintain high levels of quality. The screening process can only be successful if it is followed by timely and appropriate management of screen-detected lesions.

In essence, the management of screen-detected adenomas and carcinomas does not differ, stage for stage, from that required for symptomatic disease. However, screening detects a different spectrum of disease compared with that diagnosed in the symptomatic population (i.e. higher proportion of early disease). Thus, there are some considerations in the management of screen-detected disease that should be emphasised. In this chapter of the Guidelines the management of endoscopically detected pre-malignant lesions, pT1 cancers, as well as colon cancer and rectal cancer which is not limited to the submucosa are dealt with separately and discussion is focused on issues pertinent to screening. For these reasons, adjuvant chemotherapy and the management of advanced disease are not discussed.

Of prime general importance is the wide consensus that colorectal neoplasia is best managed by a multi-disciplinary team. The relevant disciplines include: surgery, endoscopy, pathology, radiology, radiotherapy, medical oncology, specialist nursing, genetics and palliative care [29], which should work in close collaboration with primary care. Furthermore, it is recognised that the interval between the diagnosis of screen-detected disease and the start of definitive management is a time of anxiety for the patient and affords the opportunity, if prolonged, for disease progression. For these reasons, standards have been set which aim at minimising delay [24]. Also of relevance in this regard is the recognition that colonoscopy is not merely a diagnostic procedure, but has therapeutic capacity [8], and it is essential that the endoscopist carrying out screening colonoscopy has the necessary expertise to remove all but the most demanding lesions (see also Chapter $5[32])$.

\section{Chapter 9 - Colonoscopic surveillance following} adenoma removal

Chapter 9 [3] includes 24 graded recommendations and a comprehensive strategy for surveillance after removal of adenomas in people taking part in screening programmes in any Member State. The recommendations in the EU Guidelines recognise that people with previous adenomas are at increased risk for recurrent adenomas and thus eventually colorectal cancer [5]. The risk depends mainly on findings during baseline colonoscopy, in particular the number, size and histological grade of removed adenomas. This allows categorisation of patients into different risk groups. The indication and interval for surveillance is determined primarily by the presumed risk for recurrence of advanced adenomas and cancer, and secondarily by age, co-morbidity, and patient wishes.

The primary aims of colonoscopic surveillance are to reduce the morbidity and mortality from colorectal cancer by removing high risk adenomas before they have had a chance to become malignant, and by detecting invasive cancers at an early, curable, stage. It must be kept in mind however, that colonoscopy is a costly, invasive and scarce resource. Therefore, colonoscopy surveillance should be undertaken only in people at increased risk, and at a minimum frequency required to provide adequate protection against the development of cancer. If colonoscopy surveillance is undertaken, it should be performed to the highest standard. 
Table 1 Summary Table of performance standards in colorectal cancer screening

\begin{tabular}{|c|c|c|c|}
\hline \multicolumn{2}{|c|}{ Indicator $^{1}$} & \multirow{2}{*}{$\begin{array}{l}\text { Acceptable level } \\
95 \%\end{array}$} & \multirow{2}{*}{$\begin{array}{l}\text { Desirable level } \\
>95 \%\end{array}$} \\
\hline 1 & Invitation coverage ${ }^{\text {Rec } 3.7 ; \text { Sect 3.3.1 }}$ & & \\
\hline 2 & Uptake rate Rec $3.8 ;$ Sect 3.3.1 $^{2}$ & $>45 \%$ & $>65 \%$ \\
\hline 3 & Rate of inadequate FOBTRec 3.9; 4.21; Sect 3.3.2; 4.3.4 & $<3 \%$ & $<1 \%$ \\
\hline 4 & Maximum time between test and receipt of result should be 15 days ${ }^{\operatorname{Rec} 3.15 ; \operatorname{Sect} 3.3 .4}$ & $>90 \%$ & \\
\hline 5 & Rate of referral to follow-up colonoscopy after positive test ${ }^{\text {Rec } 3.10 ; \text { Sect 3.3.2, 3.3.3 }}$ & $90 \%$ & $>95 \%$ \\
\hline 6 & $\begin{array}{l}\text { Maximum time between referral after positive screening (any modality) and follow-up } \\
\text { colonoscopy should be } 31 \text { days }\end{array}$ & $>90 \%$ & $>95 \%$ \\
\hline 7 & Compliance with follow-up colonoscopy after any positive screening test ${ }^{\text {Rec } 3.14 ; \text { Sect 3.3.2. 3.3.3 }}$ & $85 \%$ & $>90 \%$ \\
\hline 8 & $\begin{array}{l}\text { Rate of complete colonoscopies. Follow-up and screening colonoscopies to be recorded } \\
\text { separatelyRec 3.11; Rec 5.41, Sect 3.3.2. 3.3.3., 5.4.5.1 }\end{array}$ & $>90 \%$ & $>95 \%$ \\
\hline 9 & $\begin{array}{l}\text { Time interval between positive colonoscopy/FS and definitive management should be } \\
\text { within } 31 \text { days }{ }^{\text {Rec } 3.17,8.2 ; \text { Sect 3.3.4, 8.2 }}\end{array}$ & $>95 \%$ & \\
\hline 10 & $\begin{array}{l}\text { Endoscopists participating in a CRC screening programme should perform a minimum } \\
\text { no. of procedures per year }{ }^{\text {Rec } 5.38 ; \text { Sect } 5.4 .5 .1}\end{array}$ & 300 & $>300$ \\
\hline 11 & $\begin{array}{l}\text { Biopsies and lesions identified in the screening programme and the subsequent resection } \\
\text { specimen should be reported on a proforma }{ }^{\text {Rec } 7.11 ; \text { Sect 7.6.5.2.7.8 }}\end{array}$ & $>90 \%$ & \\
\hline 12 & $\begin{array}{l}\text { Rate of high-grade neoplasia reported by pathologists in a colonoscopy screening } \\
\text { programme }\end{array}$ & $<5 \%$ & \\
\hline 13 & $\begin{array}{l}\text { Rate of high-grade neoplasia reported by pathologists in a FOBT screening } \\
\text { programme }\end{array}$ & $<10 \%$ & \\
\hline
\end{tabular}

Sect (superscript) refers to the section/s of the Guidelines dealing with the respective indicator. *

$\operatorname{Rec}$ (superscript) refers to the number of the corresponding recommendation in the Guidelines. *

*The first digit of the section numbers and recommendation numbers refers to the respective chapter in the guidelines. For Chapters 1 to 10 see: $[15,18,23,11,32,31,27,30,3,6]$ respectively.

Because surveillance colonoscopy consumes considerable endoscopic resources it may prevent a country that has difficulty meeting demand from sustaining reasonable waiting times. Screening programmes should therefore have a policy on surveillance with a hierarchy of action for different risk groups based on resource availability. The policy may limit surveillance to the high risk group if sufficient resources are not available to include people with lower risk.

\section{Chapter 10 - Communication}

Chapter 10 [6] provides 35 recommendations dealing with communication in CRC screening. The large body of guidance reflects the essential goal of CRC screening programmes that is to reduce the burden of illness and death due to colorectal cancer. Screening programmes can only be successful if they ensure that as many people in the target population as possible receive the relevant information to be able to make informed decisions about whether or not they wish to attend CRC screening. As adverse effects are intrinsic to screening practice, participants should understand that a balance exists between benefits and harms associated with CRC screening [13]. A key component of CRC screening programmes, therefore, is the information and education provided about CRC, and CRC screening tests and procedures.

The recommendations in the EU Guidelines reflect the wide consensus that people who use CRC screening services should receive accurate and accessible information that reflects the most current evidence about the CRC screening test and its potential contributions to reducing illness as well as information about its risks and limitations. Achieving this goal is challenging, due to the complexity of CRC screening programmes compared to other established programmes such as screening for breast or cervical cancer. In CRC screening multiple tests are currently in use (FOBT in most, as well as flexible sigmoidoscopy (FS) and colonoscopy in some Member States). Furthermore, some screening tests are invasive, and have known adverse effects. Finally, some CRC screening procedures are generally undertaken without supervision from a healthcare professional (FOBT screening test and bowel cleansing procedure in preparation for follow-up colonoscopy or endoscopy screening). Therefore specific instructions on how to use the FOBT kit or perform the bowel cleansing procedure need to be communicated to the patient.

The recommendations in the chapter on Communication have therefore been developed to give people involved in providing and/or managing CRC screening (e.g. managers, decision-makers, health professionals etc.) an insight into the complexity of communication in CRC screening and its related critical issues. Pragmatic recommendations are also provided on information strategies/tools/interventions that can be used in current or future programmes. These recommendations mainly refer to an organised (and centralised) CRC screening programme, as this represents the gold standard to achieve (see Chapters 1 [15] and 2 [18]). In the Communication chapter, the authors specifically provide guidance for screening programmes based on the primary screening test recommended by the EU, the faecal occult blood test (FOBT, see Chapter 4 [11]) which is also the most frequently used test in programmes implemented by the Member States. Most of the recommendations can be applied to endoscopy programmes as well.

\section{Performance standards}

- Table 1 presents the performance standards in the first edition of these Guidelines. The numbering is not indicative of importance; more complete information regarding definition and context is provided in the sections indicated. As explained in the Guidelines, programmes should monitor numerous additional parameters in order to maintain and continuously improve quality. The standards listed in the present Summary Table are based on an overview of performance measurements currently available from published trial results and population-based screening programmes (see Chapter 3 [23]). In light of this evidence and experience in implementation of population based screening programmes, the authors and editors of the current version of the 
Guidelines were able to reach a consensus on the recommended targets across the EU. On occasions we have had to accept that different disciplines and different Member States show some variation of priorities and target levels. In all cases we have attempted to list what we regard as the most generally appropriate professionally agreed levels for usage in a pan-European setting. In any case, all targets should be constantly reviewed in the light of experience and revised accordingly with regard to results achieved and best clinical practice. As far as possible, targets given refer to men and women aged 50-74 years invited to and/or attending a CRC screening programme.

\section{Conclusions \\ $\nabla$}

In a multidisciplinary process, wide consensus has been achieved on a comprehensive package of evidence-based recommendations for quality assurance in colorectal cancer screening and diagnosis. Following these recommendations has the potential to enhance the control of colorectal cancer in Europe and elsewhere through improvement in the quality and effectiveness of the screening process that extends from systematic invitation to management of screen-detected cases.

\section{Disclaimer \\ $\nabla$}

The views expressed in this document are those of the authors. Neither the European Commission nor any person acting on its behalf can be held responsible for any use that may be made of the information in this document.

\section{Competing interests: No competing interests reported}

\section{Acknowledgements}

$\nabla$

The comments and suggestions received from consultation of the European Cancer Network and international experts are gratefully acknowledged. Contributions during the main phase of drafting and revising individual chapters are acknowledged separately in the respective articles. Comments and suggestions received during the consultation phase prior to publication of the guidelines were provided by the following contributors:

N. Antoljak, Croatia; S. Arrossi, Argentina; J. Blom, Sweden; M. Dai, China; J. Daniel, IARC; M. de Camargo Cancela, IARC; N. Delicata, Malta; S.Ducarroz, IARC; H. Erfkamp, Austria; L. Faulds Wood, United Kingdom; S. Frkovic-Grazio, Slovenia; G. Grazzini, Italy; J. Green, United Kingdom; C. Hamashima, Japan; C. Herrmann, IARC; M. Filip Kaminski, Poland; W. C. Lee, Korea; T. Lignini, IARC; E. Lucas, IARC; S. Mádai, Hungary; J. C. Marinho, Portugal; A. Morais, Portugal; R. Muwonge, IARC; M. Nadel, USA; L. Neamtiu, Romania; M. Pignone, USA; J. V. Psaila, Malta; J. Ren, IARC; G. Rennert, Israel; V. J. L. Rodrigues, Portugal; C. Sauvaget, IARC; A. Scharpantgen, Luxembourg; M. Siddiqi, India; D. Sighoko, IARC; R. Smith, USA; S.Suchanek, Czech Republic; E. Suonio, IARC; W.-M. Tong, China; L. Voti, IARC; J. Maučec Zakotnik, Slovenia; V Zaksas, Lithuania.

The production of the Guidelines was supported by the European Union through the EU Public Health Programme, (grant agreement no. 2005317: Development of European Guidelines for Quality Assurance of Colorectal Cancer Screening). Partner insti- tutions: Oxford University Cancer Screening Research Unit, Cancer Epidemiology Unit, University of Oxford, Oxford, United Kingdom; Unit of Cancer Epidemiology, Centre for Cancer Epidemiology and Prevention (CPO) and S.Giovanni University Hospital, Turin, Italy; Public Association for Healthy People, Budapest, Hungary; European Cancer Patient Coalition (ECPC), Utrecht, Netherlands ; Quality Assurance Group, Section of Early Detection and Prevention, International Agency for Research on Cancer, Lyon, France.

Financial support was also received through the Public Affairs Committee of the United European Gastroenterology Federation, and from a cooperative agreement between the American Cancer Society and the Division of Cancer Prevention and Control at the Centers for Disease Control and Prevention.

\section{References}

1 Arbyn $M$, Anttila A, Jordan $J$ et al. European Guidelines for Quality Assurance in Cervical Cancer Screening. Second edition - summary document.. Ann Oncol 2010; 21: $448-458$

2 Arbyn M, Anttila A, Jordan J et al. (eds.) European guidelines for quality assurance in cervical cancer screening - second edition. Luxembourg: European Comission, Office for Official Publications of the European Communities; 2008

3 Atkin $W$, Valori R, Kuipers EJ et al. European guidelines for quality assurance in colorectal cancer screening and diagnosis. First Edition. Colonoscopic surveillance following adenoma removal. Endoscopy 2012; 44: SE151 -SE163

4 Atkin WS, Edwards R, Kralj-Hans I et al. Once-only flexible sigmoidoscopy screening in prevention of colorectal cancer: a multicentre randomised controlled trial. Lancet 2010; 375: 1624-1633

5 Atkin WS, Morson BC, Cuzick J. Long-term risk of colorectal cancer after excision of rectosigmoid adenomas. N Engl J Med 1992; 326: 658 - 662

6 Austoker J, Giordano L, Hewitson P et al. European guidelines for quality assurance in colorectal cancer screening and diagnosis. First Edition. Communication. Endoscopy 2012; 44: SE164-SE185

7 Commission of the European Communities. Report from the Commission to the Council, the European Parliament, the European Economic and Social Committee and the Committee of the Regions - Implementation of the Council Recommendation of 2 December 2003 on cancer screening (2003/878/EC). Brussels: 2008: $\operatorname{COM}(2008) 882$ final

8 Cotton $P B$, Williams $C B$. Colonoscopic polypectomy and therapeutic procedures. Practical Gastrointestinal Endoscopy. 4th: Edition Blackwell Science; 1996: 275-302

9 Council of the European Union. Council Recommendation of 2 December 2003 on cancer screening (2003/878/EC). Off J Eur Union; 2003: $34-38$

10 Ferlay J, Shin HR, Bray F et al. GLOBOCAN 2008, Cancer Incidence and Mortality Worldwide: IARC CancerBase No. 10 [Internet]. Lyon, France: International Agency for Research on Cancer; 2010: Accessed 6/4/ 2012

11 Halloran S, Launoy G, Zappa M. European guidelines for quality assurance in colorectal cancer screening and diagnosis. First Edition. Faecal Occult Blood Testing. Endoscopy 2012; 44: SE65-SE87

12 Hardcastle JD, Chamberlain JO, Robinson MH et al. Randomised controlled trial of faecal-occult-blood screening for colorectal cancer. Lancet 1996; 348: $1472-1477$

13 Holland W, Stewart S, Masseria C. Policy Brief: Screening in Europe. Copenhagen: WHO Regional Office; 2006

14 Kronborg $O$, Fenger $C$, Olsen J et al. Randomised study of screening for colorectal cancer with faecal-occult-blood test. Lancet 1996; 348: $1467-1471$

15 Landsorp-Vogelaar I, von Karsa L. European guidelines for quality assurance in colorectal cancer screening and diagnosis. First Edition. Introduction. Endoscopy 2012; 44: SE15-SE30

16 Leslie A, Carey FA, Pratt NR et al. The colorectal adenoma-carcinoma sequence. Br J Surg 2002; 89: 845-860

17 Levin B, Lieberman DA, McFarland B et al. Screening and surveillance for the early detection of colorectal cancer and adenomatous polyps, 2008: a joint guideline from the American Cancer Society, the US Multi-Society Task Force on Colorectal Cancer, and the American College of Radiology. Gastroenterology 2008; 134: 1570-1595 
18 Malila N, Senore C, Armaroli P. European guidelines for quality assurance in colorectal cancer screening and diagnosis. First Edition. Organisation. Endoscopy 2012; 44: SE31 - SE48

19 Mandel JS, Church TR, Bond JH et al. The effect of fecal occult-blood screening on the incidence of colorectal cancer. N Engl J Med 2000; 343: $1603-1607$

20 Mandel JS, Church TR, Ederer F et al. Colorectal cancer mortality: effectiveness of biennial screening for fecal occult blood. J Natl Cancer Inst 1999; 91: 434-437

21 Minozzi S, Armaroli P, Banzi R et al. European guidelines for quality assurance in colorectal cancer screening and diagnosis. First edition. Appendix 1: Systematic evidence review. 2010: http://bookshop.europa.eu/en/european-guidelines-for-quality-assurance-in-colorectalcancer-screening-and-diagnosis-pbND3210390/ Accessed 11/2/2012

22 Minozzi S, Armaroli P, Segnan N. European guidelines for quality assurance in colorectal cancer screening and diagnosis. First edition. Principles of evidence assessment and methods for reaching recommendations. Endoscopy 2012; 44: SE9-SE14

23 Moss $S$, Ancelle-Park $R$, Brenner $H$. European guidelines for quality assurance in colorectal cancer screening and diagnosis. First edition. Evaluation and interpretation of screening outcomes. Endoscopy 2012; 44: SE49-SE64

24 NHS. Bowel Screening Programme Clinical Standards. Scotland: NHS Quality Improvement; 2007: http://www.bowelscreening.scot.nhs. uk/wp-content/uploads/2007/06/bowelsc_stnf_feb07.pdf Accessed $11 / 2 / 2012$

25 Perry $N$, Broeders $M$, de Wolf $C$ et al. European guidelines for quality assurance in breast cancer screening and diagnosis. Fourth edition summary document. Ann Oncol 2008; 19: 614-622

26 Perry $N$, Broeders $M$, de Wolf $C$ et al. (eds.) European guidelines for quality assurance in breast cancer screening and diagnosis. Fourth edition. Luxembourg: Office for Official Publications of the European Communities; 2006

27 Quirke P, Risio M, Lambert R et al. European guidelines for quality assurance in colorectal cancer screening and diagnosis. First edition. Quality assurance in pathology in colorectal cancer screening and diagnosis. Endoscopy 2012; 44: SE116-SE130

28 Segnan $N$, Patnick $J$, von Karsa $L$ (eds.) European guidelines for quality assurance in colorectal cancer screening and diagnosis. First edition. Luxembourg: European Commission, Publications Office of the European Union; 2010

29 SIGN. Scottish Intercollegiate Guidelines Network - Guidelines for the management of colorectal cancer. 2003: http://www.sign.ac.uk/pdf/ sign67.pdf Accessed 11/2/2012

30 Steele RJC, Pox C, Kuipers E et al. European guidelines for quality assurance in colorectal cancer screening and diagnosis. First edition. Management of lesions detected in colorectal cancer screening. Endoscopy 2012; 44: SE140 - SE150

31 Steele RJC, Rey J-F, Lambert R. European guidelines for quality assurance in colorectal cancer screening and diagnosis. First edition. Professional requirements and training. Endoscopy 2012; 44: SE106-SE115

32 Valori $R$, Rey J-F, Atkin $W$ et al. European guidelines for quality assurance in colorectal cancer screening and diagnosis. First edition. Quality assurance in endoscopy in colorectal screening and diagnosis. Endoscopy 2012; 44: SE88-SE105

33 Vieth $M$, Quirke $P$, Lambert $R$ et al. European guidelines for quality assurance in colorectal cancer screening and diagnosis. First edition. Annex - Annotations of colorectal lesions. Endoscopy 2012; 44: SE131-SE139

34 von Karsa L, Anttila A, Ronco G et al. Cancer Screening in the European Union. Report on the implementation of the Council Recommendation on Cancer Screening - First Report. Luxembourg: European Commission; 2008: http://ec.europa.eu/health/archive/ph_determinants/genetics/documents/cancer_screening.pdf Accessed 11/2/2012

35 von Karsa L, Lignini TA, Patnick J et al. The dimensions of the CRC problem. Best Pract Res Clin Gastroenterol 2010; 24: 381 - 396

36 Wilson JM, Jungner YG. Principles and practice of mass screening for disease. World Health Organization; 1968: http://whqlibdoc.who.int/ php/WHO_PHP_34.pdf Accessed 11/2/2012 